\title{
Travelling ionospheric disturbance properties deduced from Super Dual Auroral Radar measurements
}

\author{
J. W. MacDougall", D. A. Andre, G. J. Sofko, C.-S. Huang, A. V. Koustov \\ Institute of Space and Atmospheric Studies, University of Saskatchewan, Saskatoon, Saskatchewan, Canada \\ * On sabbatical from Department Electrical Engineering, University of Western Ontario, London, Ontario, Canada
}

Received: 14 April 2000 / Revised: 21 August 2000 / Accepted: 12 September 2000

\begin{abstract}
Based on modeling of the perturbations in power and elevation angle produced by travelling ionospheric disturbances (TIDs), and observed by the Super Dual Auroral Radar Network, procedures for determining the TID properties are suggested. These procedures are shown to produce reasonable agreement with those properties of the TIDs that can be measured from simultaneous ionosonde measurements. The modeling shows that measurements of angle-of-elevation perturbations by SuperDARN allows for better determination of the TID properties than using only the perturbations of power as is commonly done.
\end{abstract}

Key words: Ionosphere (auroral ionosphere; ionosphere-atmosphere interactions)

\section{Introduction}

It was shown by Samson et al. (1989) that the fringes of echo power that were often observed on the ground scatter returns observed by the Super Dual Auroral Radars (SuperDARN) were caused by gravity waves that modified the ionospheric electron density contours so that they caused focusing of the radio waves. These perturbations of the ionospheric density are usually referred to as travelling ionospheric disturbances (TIDs). Since the initial identification of TIDs as the cause of the SuperDARN ground scatter signal strength perturbations, there have been many TID studies making use of the extensive SuperDARN data archive. While SuperDARN is not an ideal instrument for studying TIDs, since it does not directly measure any TID property, nevertheless the very obvious TIDassociated radar echo enhancements and the very extensive coverage of the SuperDARN radars in both

Correspondence to: J. W. MacDougall

e-mail: jmacdoug@julian.uwo.ca space and time makes it attractive to pursue TID studies using the SuperDARN data archives.

A further attraction of using SuperDARN data to study TIDs is that the radars are configured primarily to observe the auroral zone, which is known to be the most important source of TIDs (see review by Hunsucker, 1982). Many researchers view the TIDs propagating equatorward from auroral latitudes as signatures of processes that are taking place in auroral regions. At this time some of the processes that can create gravity waves, and hence TIDs, such as Joule heating and Lorentz forcing associated with auroral electrojets are well understood (see review by Hocke and Schlegel, 1996), while other processes such as the gravity wave amplification in convecting plasma proposed by Robinson (1994) are not yet verified. Thus our developing understanding of the processes that lead to TIDs make studies of the TIDs attractive as ways of detecting processes that are taking place in auroral latitudes.

The early TID studies using SuperDARN data (Bristow et al., 1994) simply assumed that, for low amplitude TIDs, the TID is located at close to half the range of the ground backscatter reflection distance. Thus the velocity of the TID would be about half the velocity determined from the time variation of distance to the backscatter intensity maximum produced by the focusing action of the TID. This assumption that $1 / 2$ is an appropriate factor when making TID velocity calculations was challenged by Hall et al. (1999) who found, using simple raytracing analysis of TIDs, that $1 / 1.66$ is a more suitable factor for TID velocity calculations. In this present study, we perform more extensive raytracing analysis of TID focusing and show that the appropriate proportionality factor can be close to 1.0. We also show that using SuperDARN angle-of-arrival measurements in combination with the amplitude measurements leads to improvements in the determination of the TID properties.

\section{TID simulations}

The TID modeling that will be presented uses as its 'typical' example the TID produced intensity and angle- 
of-arrival variations on one particular day as observed by the Saskatoon SuperDARN radar. The portion of this day when the TIDs were seen is shown in Plate 1. The plate shows both the intensity variations (top panel) and angle-of-arrival variations (bottom panel). This particular day was chosen mainly because the TID variations were clearly defined. Also we chose a day when there were ionosonde measurements of the TIDs (see later).

The TIDs on the day shown in Plate 1 cause the forward sloping features at about $1100-1500 \mathrm{~km}$ range. These echoes are classified as ground scatter based on their very low velocities and narrow spectral widths. Plate 1 also shows ionospheric backscatter from near $(<1000 \mathrm{~km})$ ranges at the left and right sides of the panels, and far range ionospheric backscatter at around $2500 \mathrm{~km}$ during most of this interval. It may be noted that the TIDs appear to be also modulating the intensity and angles-of-arrival of the far range ionospheric backscatter.
The aim of our modeling is to produce patterns of intensity and angle-of-arrival that visually resemble those shown in Plate 1 for the ground scattered echoes. Since most TID deductions from the SuperDARN measurements simply make measurements from patterns such as those shown in Plate 1, if we can reproduce these patterns from our modeling then we can attempt to determine the best way of deducing the TID properties from these observed patterns.

As mentioned earlier, we had ionosonde measurements from the Canadian Advanced Digital Ionosonde at Rabbit Lake which is located at a range of $680 \mathrm{~km}$ under the SuperDARN beam (5), data from which are displayed in Plate 1. This ionosonde was recording ionograms once each minute, and $3 \mathrm{MHz}$ and $4 \mathrm{MHz}$ 'fixed frequency' samples each $30 \mathrm{~s}$. From the ionograms we determined the ionospheric electron density profile by manually scaling a few ionograms and using POLAN
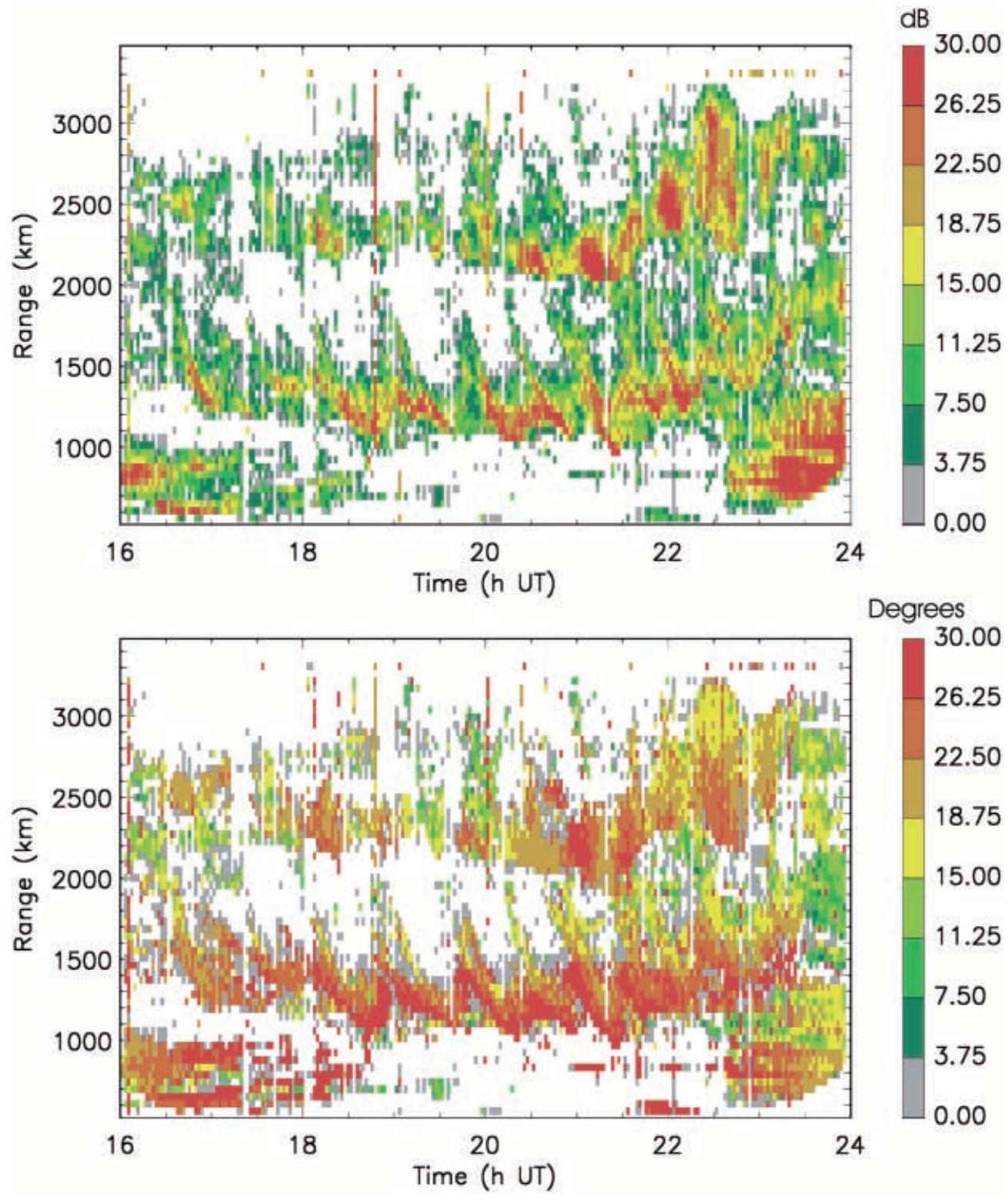

Plate 1. Range-time-intensity plot (top panel), and range-timeelevation angle plot (bottom panel) for daytime part of December 6, 1997. Midday is about 19:00 UT. The TID perturbations are the series of sloping streaks at about $1100-1500 \mathrm{~km}$ range 
(Titheridge, 1985). Figure 1 shows some of the results along with a simplified electron density profile that was used in the simulations. The F layer has a peak electron density near $7 \times 10^{11}$ (plasma frequency $\sim 8 \mathrm{MHz}$ ) near $240 \mathrm{~km}$, and a relatively flat bottomside near $200 \mathrm{~km}$. Densities below $200 \mathrm{~km}$ in the $\mathrm{E}$ region were relatively low. For purposes of modeling, as shown in Fig. 1, we approximated the bottomside electron density profile with a mathematical simple cosine function with peak electron density $7 \times 10^{11} \mathrm{el} / \mathrm{m}^{3}$ at $240 \mathrm{~km}$, reducing to density 0 at $190 \mathrm{~km}$. The weak E layer was not modeled since we could not accurately determine its properties and it should have very little effect at the $13 \mathrm{MHz}$ SuperDARN frequency.

The TIDs could be easily seen on the virtual height versus time plots from the two fixed frequencies. The TIDs had typical peak-to-peak height variations of about $10 \mathrm{~km}$. The height peaks occurred earlier on the $4 \mathrm{MHz}$ plots than on the $3 \mathrm{MHz}$ plots, as expected for TIDs which have downward phase propagation. From the difference in reflection heights of the two frequencies, as determined from electron density profiles, and the time difference for TID features on the two frequencies, we determined that the vertical wavelength was about $100 \mathrm{~km}$ at $210 \mathrm{~km}$ height. We used this vertical wavelength in most of our TID modeling, and assumed it to be constant since reflections were from a limited height range on the bottomside of the $\mathrm{F}$ region. The other TID properties used in our modeling were amplitudes of 5, 10, and $15 \mathrm{~km}$; and horizontal wavelengths of $250,350,500$, and $750 \mathrm{~km}$. The typical period of the gravity waves on this day was about $40 \mathrm{~min}$. The gravity wave properties that were used in our modeling are very typical for gravity waves at about $225 \mathrm{~km}$ (see figure on p 288 of Hines, 1974). Of course the period of $40 \mathrm{~min}$ is not meaningful if these waves are actually coming from separate source excitation events: it is

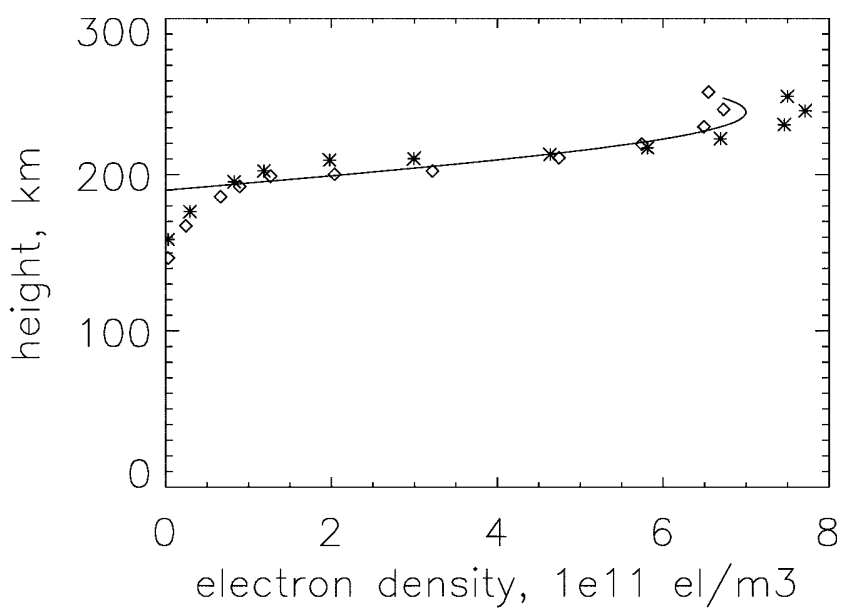

Fig. 1. Measured and modeled electron density profiles. The electron densities as derived from the CADI measurements are shown by the asterisks for time 2000 UT (time of a height maximum of the TIDs), and by the diamond symbols for 2030 UT (height minimum of the TIDs). The modeled cosine bottomside electron density profile is shown by the solid curve merely the time interval between events. However the ionosonde height-versus-time plots showed reasonably sinusoidal patterns with $\sim 40$ min periods so we take $40 \mathrm{~min}$ as being meaningful for these TIDs.

In our modeling we merely had the gravity waves vertically displacing the electron density contours: We assumed that they did not vary the electron density. This is inconsistent since, if the waves have finite vertical wavelength, the density should vary as $\frac{\partial N}{\partial t}=-\nabla N \overrightarrow{\mathrm{V}}$, where $N$ is the electron density and $\vec{V}$ is the velocity due to the gravity waves. This equation can be simplified if it assumed that the $\mathrm{F}$ region ionization is constrained to stay on the same magnetic field line. As mentioned, we did not vary the ionization as implied by this equation since, (a) we wanted to model the effects of a purely sinusoidal height perturbation due to the gravity waves, and (b) we found that the density variations shown by the Rabbit Lake ionosonde were relatively small. The ionosonde data were used to examine the peak ionospheric electron density during this event. Before 20:00 UT there were peak-to-peak density variations of about $1 \mathrm{MHz}$ in plasma frequency associated with the early gravity wave cycles, but after 20:00 UT when Plate 1 shows better defined waves the perturbations of peak electron density were somewhat smaller $(<15 \%)$. For these reasons our modeling did not vary the electron densities, but merely displaced them vertically due to the gravity waves. The electron density varied slowly throughout this day, rising from $\sim 50 \times 10^{11} \mathrm{el} \mathrm{m}^{-3}$ around 1730 UT to the peak value shown in Fig. 1 $\left(\sim 8 \times 10^{11} \mathrm{el} \mathrm{m}^{-3}\right)$ at local midday, and then decreasing gradually back to $\sim 5 \times 10^{11} \mathrm{el} \mathrm{m}^{-3}$ around $2230 \mathrm{UT}$.

Modeling results for $13 \mathrm{MHz}$ radar frequency are illustrated by Fig. 2. The top panel in this figure shows (dashed) the ionospheric contours each $1.0 \times 10^{11} \mathrm{el} /$ $\mathrm{m}^{-3}$. The perturbations due to the gravity waves, which for this simulation had amplitude $10 \mathrm{~km}$ and horizontal wavelength $500 \mathrm{~km}$, are clearly seen. Rays from the radar at the origin are shown for each $1^{\circ}$ step in elevation (actually the simulation used $0.05^{\circ}$ elevation steps, only the $1^{\circ}$ steps are plotted in this panel). Raytracing used the procedure derived by Hazelgrove (1963). Only the ordinary ray was traced since it was assumed that for these daytime results the extraordinary wave would be relatively weaker due to absorption. The magnetic field was kept constant at its $680 \mathrm{~km}$ range value (Rabbit Lake). The rays start at $0^{\circ}$ elevation (most

Fig. 2. Simulation results at one particular time (phase) of the TID. In the top panel the electron density contours are shown for each $1 \times 10^{11} \mathrm{el} / \mathrm{m}^{3}$ (up to the electron density peak). The TID properties are: amplitude $10 \mathrm{~km}$, horizontal wavelength $500 \mathrm{~km}$, vertical wavelength $100 \mathrm{~km}$. The raypaths are shown in $1^{\circ}$ elevation angle steps starting from $0^{\circ}$ (furthermost clockwise). The curved raypaths below the ionosphere are due to Earth curvature. The second panel shows the strength of each raypath (the simulation stepped the elevation angle in $1 / 20^{\circ}$ steps). Note that the horizontal scale in the top panel is ground distance whereas the horizontal scale for the lower panels is raypath range. Panel 3 shows the echo strength for each $45 \mathrm{~km}$ range bin (see text for details of this calculation). The bottom panel shows the path lengths versus elevation angle (solid), and the weighted average binned elevation angles (dotted) 


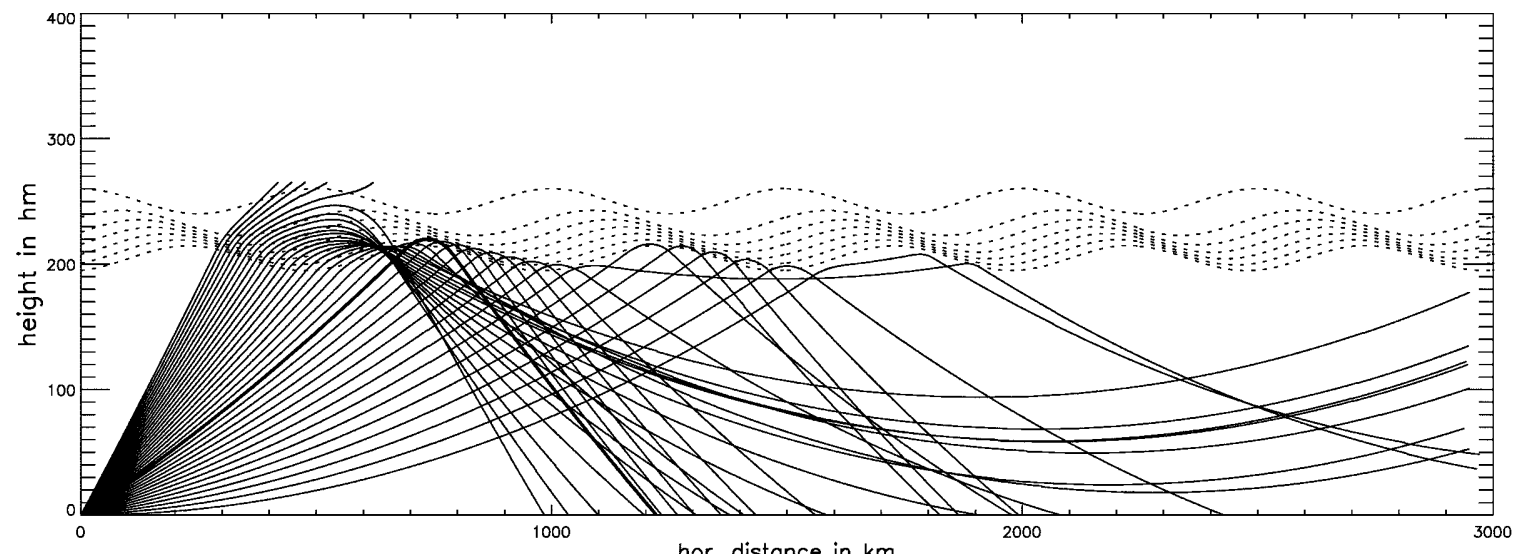

hor. distance in $\mathrm{km}$
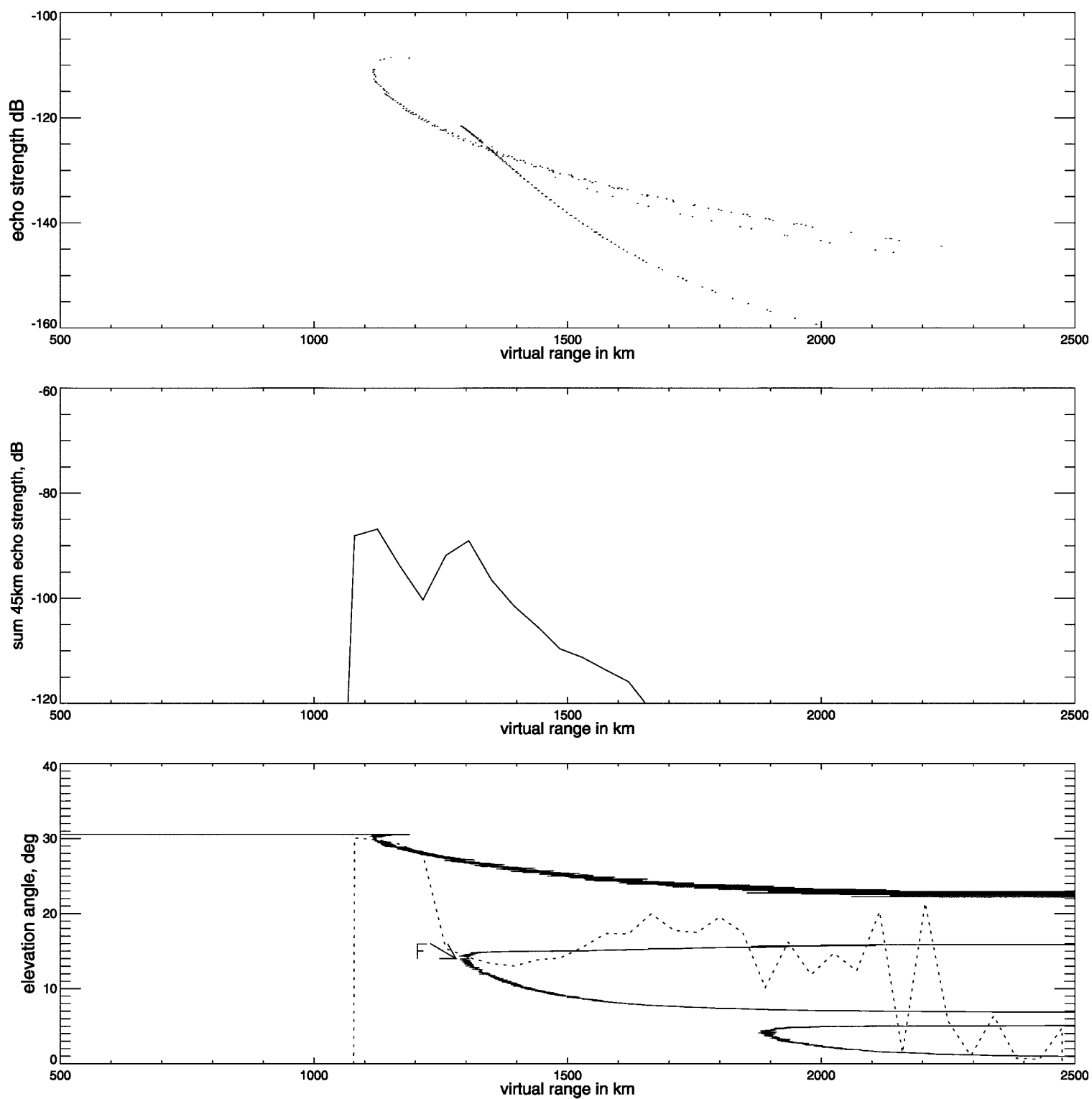

clockwise rays) and appear to curve upwards due to Earth curvature. These low angle rays are reflected to far ranges. Rays at higher elevation angles mostly strike the ground after reflection, although a few are reflected to remote ranges. At high elevation angles some of the rays
(Pedersen rays) travel significant distances more-or-less horizontally through the ionosphere. At higher elevation angles (most counterclockwise rays) the rays are not reflected (tracing of the rays stopped if they propagated through the F region peak). The rays that are 'focused' 
by the TIDs can be most clearly seen at the reflections above about $1400 \mathrm{~km}$ from the $3 \mathrm{rd}$ cycle of the TID. It is the peak and downward sloping part of the TID that is associated with the focusing.

The focusing effects are more clearly revealed by the lower panels of Fig. 2. In the second panel the strength versus range for the various rays are shown. The range is calculated from the raypath and the group speed until the rays strike the ground. The strength that is shown in this panel is the strength back at the radar of two-way travel along the same raypath and includes effects due to the antenna pattern, path distance, and ground scatter.

Our first simulations assumed, as for most previous simulations, that the ground backscattered ray returned along the same path as the 'forward' ray. We then summed the power for all the rays that had pathlengths quantized to the $45 \mathrm{~km}$ range bins used by SuperDARN. Similarly we determined the angle for each range bin by an amplitude weighted average of the angles-of-arrival of the rays associated with that particular range bin.

These initial simulations (not shown) that used the assumption that the return raypath is the same as the forward raypath did give a reasonable reproduction of the observed patterns of power and angle-of-arrival variations due to the TIDs. However there were minor inconsistencies between the simulated patterns and the observed patterns. We discovered that better simulations could be produced if we made the more realistic assumption that the radio waves are scattered at the ground and can travel back to the radar along any raypath provided that the backscattered ray starts from a ground distance near to the ground distance of the forward ray. Thus in our procedure we first determined the ground distance for each of the forward raypaths. The power of these forward rays included only antenna pattern effects since the ground scatter area was assumed to be 'beamfilling' so there was no power dependency on range for these forward rays. For our calculation of the backscattered rays, for each forward ray we took a $45 \mathrm{~km}$ ground distance cell centered on where the ray struck the ground, and identified all the forward raypaths that fell into this ground distance cell. We assumed that the ground could backscatter energy to the radar along any of these raypaths. The $45 \mathrm{~km}$ ground distance cell size (same size as the SuperDARN range cells) is somewhat arbitrary since one merely wants cells that are compatible with the resolution of the simulation; cell sizes should be large enough that the ground distances for a few raypaths will fall into any given cell. Having identified all the possible scattered raypaths back to the radar, for each of these raypaths we summed the forward and return distance and determined the $90 \mathrm{~km}$ bin that the ray would be quantized into. (As with most radars, SuperDARN divides the overall distances by 2 to determine range; the $45 \mathrm{~km}$ SuperDARN range bins are actually $90 \mathrm{~km}$ total pathlength bins.) The intensity of each return ray was corrected for ground backscatter strength, return pathlength, and antenna pattern.

The antenna pattern effect applies to both outgoing and returning rays since the same antenna is used for both transmitting and receiving. The antenna was assumed to be an isotropic radiator (as a function of elevation angle) situated $15.3 \mathrm{~m}$ above a $95 \%$ reflecting ground. Thus rays at $0^{\circ}$ are almost completely cancelled by ground reflection, whereas, at $13 \mathrm{MHz}$, rays at elevation angle of $22^{\circ}$ are maximally reinforced by ground reflection. The pathlength correction applies to returning rays and is $1 /$ (range) $)^{2}$ since obviously the receiving radar antenna is not beamfilling.

The ground backscatter coefficient provided more of a quandary. Hall et al. (1999) adapted a typical backscatter formula from formulas applicable to much higher frequencies. For the present study the observed backscatter for an unperturbed ionosphere was examined. For an unperturbed ionosphere only one raypath (except for Pedersen rays) falls at each ground distance so the return path must be the same as the outgoing path. Thus the observed signal strength versus distance tells us about losses on the two-way raypath. We can compare observations with simple modeling to check the backscatter formula. This modeling assumes that we know the antenna pattern and that there is no absorption. Doing this modeling showed that the formula used in Hall et al. (1999) had backscatter that was too strong for low elevation angles. Accordingly the formula was modified for this study and for elevation angles less than $60^{\circ}$ (the angles of importance for this study) the backscatter coefficient, relative to $0 \mathrm{~dB}$ for specular reflection, was taken as $\sigma=-30.0+1.0 \times$ (elevationangle -60.0) dB.

There are theoretical formulas that predict the backscatter coefficient if one knows the statistical properties of the ground scattering region. Schanda (1986, Fig. 4.23) shows backscatter characteristics that are similar to those adopted for our simulations if the ground correlation distance is large, in terms of wavelength. The low rolling hills in the SuperDARN ground scatter region north of Saskatoon might be expected to have a large correlation distance.

In our simulations when the incident and scattered elevation angles are different the backscatter formula needs to be modified slightly. For our simulation we simply used this formula with the relevant elevation angle taken to be that of the backscattered ray.

The results of the simulation are shown in the lower panels of Fig. 2. The third panel shows the sum of the power of all the backscattered rays for each $45 \mathrm{~km}$ range bin. It can be seen that there is a secondary peak at a range of about $1300 \mathrm{~km}$ and this is the peak associated with focusing by the TID. In the bottom panel the range is shown as a function of elevation angle for the forward raypaths (solid curve). The perturbations associated with the TID show up clearly in this plot. The weighted average angles versus range are shown in this panel by the dashed curve. Since SuperDARN determines the elevation angles of the received signals by means of an interferometer, it is the weighted elevation angles at the receiver that are shown here.

The proper weighting of the angles of the parcel of returning rays is somewhat uncertain. The method that SuperDARN uses to measure the angles of the rays is to cross-correlate the signals from the main antenna array 
with a smaller interferometer array. This is referred to as "multiplicative processing". This type of angle-of-arrival measurement is discussed by Gething (1991) in his book where he presents results from a number of studies. The studies show that for two returning rays there is a "capture effect" whereby the average direction measured is that of the stronger of the two rays. No results are presented for the case of a larger number of returning rays which is probably the case for the ground reflected SuperDARN echoes. We repeated our angle calculations and plots assuming that the angle would be that of the strongest component rather than a power weighted average of the angles as used in our first simulations. The results were practically indistinguishable.

We repeated the simulations shifting the TID towards the left by $1 / 40$ of a cycle each time. These 40 simulations per cycle were then converted to contour plots of power and angle, one of which is shown in Plate 2. The time scale on the panels of this plate is shown as $40 \mathrm{~min}$ per cycle since this was typical for the observations shown in Plate 1 . It can be seen that the significant features of the observed patterns of power and elevation angle variations are being reasonably well reproduced.

We repeated the simulations for our three choices of TID amplitude $(5,10,15 \mathrm{~km})$ and four choices of horizontal wavelength $(250,350,500$, and $750 \mathrm{~km})$. Patterns similar to Plate 2 were found for all these, with of course some variation of detail. We did not find that the magnitudes of the variation of power produced by the various TIDs was sufficiently different that one could tell much about the TID properties by just looking at the magnitudes of the power variations as had been suggested in an earlier study (Bristow and Greenwald, 1995). A recent study by Stocker et al. (1999) suggested that examining the range variation of the nearest range edge of the backscatter enables one to deduce some TID properties. Our simulations did show that the magnitude of the range variation of the near edge was increased for larger amplitude TIDs. However, the typical increase was only by two range bins when the TID amplitude increased from 5 to $15 \mathrm{~km}$, and it seemed that it would be difficult to make deductions about the TID amplitudes from the near edge variations which, see Plate 1, are usually not well defined.

Our modeling does indicate that it is possible to deduce some of the TID properties from the observed power and elevation angle patterns. For each of our simulations we drew subjective best fit lines to the patterns (see dashed lines on Plate 2) and determined the apparent velocity implied by these lines. A summary of these apparent velocities is shown in Fig. 3. The labels beside each symbol on the figure indicate whether the apparent velocity (speed) was determined from the Power or Angle, and the numbers give the amplitude of the TID. The perfect fit would be the solid line. (In a simulation the exact speed is known: it is just the chosen wavelength divided by the period i.e., $40 \mathrm{~min}$.) It can be seen that the points cluster around the exact $r=1.0(r$ is the ratio of apparent speed to true speed) line. Note that the speeds determined from the elevation angles are always an underestimate, whereas the speeds from the power are always an overestimate. Also note that it is the TID amplitude relative to the horizontal wavelength that is important since for the short, $250 \mathrm{~km}$, wavelength even the low amplitude TIDs gave results close to $r=1.0$. As the amplitude of the TIDs increases, the apparent speeds move closer to the $r=1.0$ line, while for lower amplitude TIDs the speeds move away from the $r=1.0$ line. For relatively small amplitude TIDs the apparent velocities determined from the power fall close to the $r=1.66$ line inferred in the Hall et al. (1999) study. Ultimately, as the TID amplitudes approach $0 \mathrm{~km}$ the apparent velocities determined by the power would approach $r=2.0$ as assumed by Bristow et al. (1994). However, for typical TID amplitudes (5-15 km from inspection of the ionosonde data) the speeds are near to the $r=1.0$ line. At first it seems counterintuitive that the apparent speed of the observed backscatter perturbations is close to the TID speed since one would expect the backscatter range to increase faster than the movement of the TIDs. The explanation of why this does not happen is shown by Fig. 2. In the top panel of this figure it can be seen that the ionosphere-to-ground portions of the focused rays from the trailing slope of the TIDs follow more-or-less parallel paths from ionosphere to ground. As the TID moves, these ionosphereto-ground raypath portions, for the focused rays, tend to stay closely the same length. It is only the portion of the raypaths from the radar to the ionosphere that changes length, and the rate of change of these portions of the raypaths is approximately the same as the speed of the TIDs.

Therefore, as a first approximation, the TID speed is about the speed deduced from the slope of the power or angle enhancements. If the angle plots are available the speeds deduced from the elevation angle enhancements are a closer estimation than are the speeds derived from the power plots. When measuring the apparent speed from the elevation angle enhancements one must be careful to draw the apparent velocity line through the elevation angle maxima as is done in Plate $2 \mathrm{~B}$. The overall shape of the elevation angle plot is determined by the received power being above some threshold so the overall shape of the elevation angle plot will resemble the shape of the power plot, as can be clearly seen for Plate 1. In drawing the apparent velocity lines for the elevation angle plot the shape of the plot should be ignored and the line drawn through the elevation maxima.

It is possible to obtain an even better speed estimation if both the power and angle plots are available. Let us assume that the correct speed $V c$ can be deduced from the speed $V p$ calculated from the power plot and the speed $V a$ calculated from the angle plot. We assume that an equation $V c=x V p+y V a$ would apply. Minor rearrangement of this equation using $X=V p / V c$ and $Y=V a / V c$ gives, $Y=-x / y X+1 / y$, the equation of a straight line. In Fig. 4 we plot speeds calculated from our modeling normalized to $X$ and $Y$ and show a least squares fitted straight line. It can be seen that the data 

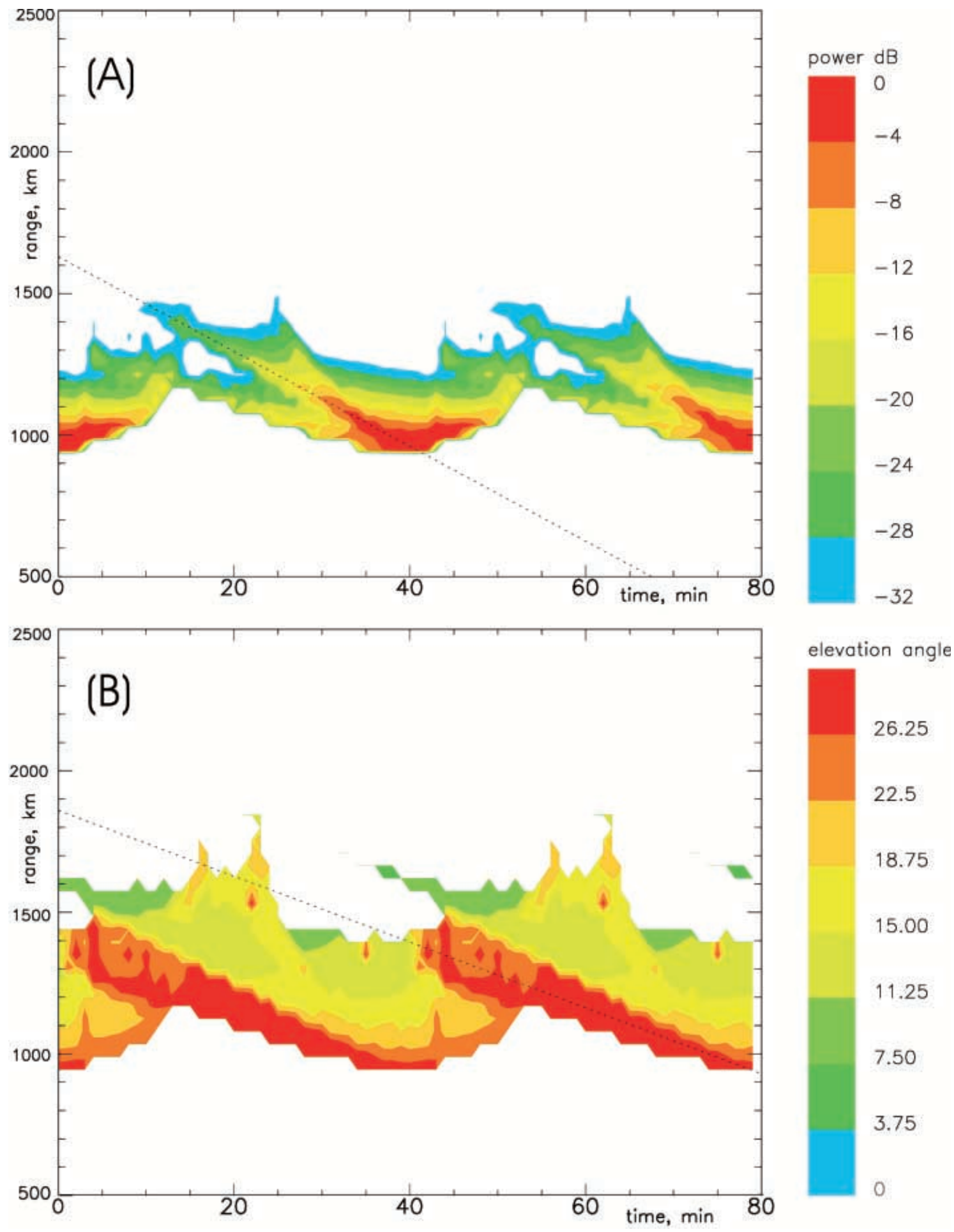

Plate 2. Simulations of $\mathbf{A}$ the range-time-intensity and $\mathbf{B}$ range-timeelevation angle plots. The ionospheric properties were: $h_{\max }=250 \mathrm{~km}, N_{\max }=7.0 \times 10^{11} \mathrm{el} / \mathrm{m}^{3}$, cosine profile going to

do have a reasonably linear distribution on the plot so the fitted line is meaningful. From the fitted line we deduce that the correct velocity can be deduced from the apparent velocities, for all TID amplitudes, as:

$V c=0.23 \mathrm{Vp}+0.84 \mathrm{Va}$
0 at $200 \mathrm{~km}$. The gravity wave properties were: $\Delta h=10 \mathrm{~km}$, $\lambda_{\text {horizontal }}=500 \mathrm{~km}, \lambda_{\text {vertical }}=100 \mathrm{~km}$, period $T=40 \mathrm{~min}$

Thus measuring both apparent velocities, $V p$ and $V a$, and using Eq. (1) should give a reasonably good speed estimation. Of course the simulations on which this estimation is based used an ionosphere that was typical for December at high latitudes and had a relatively steep bottomside. Also the vertical wavelength of the 


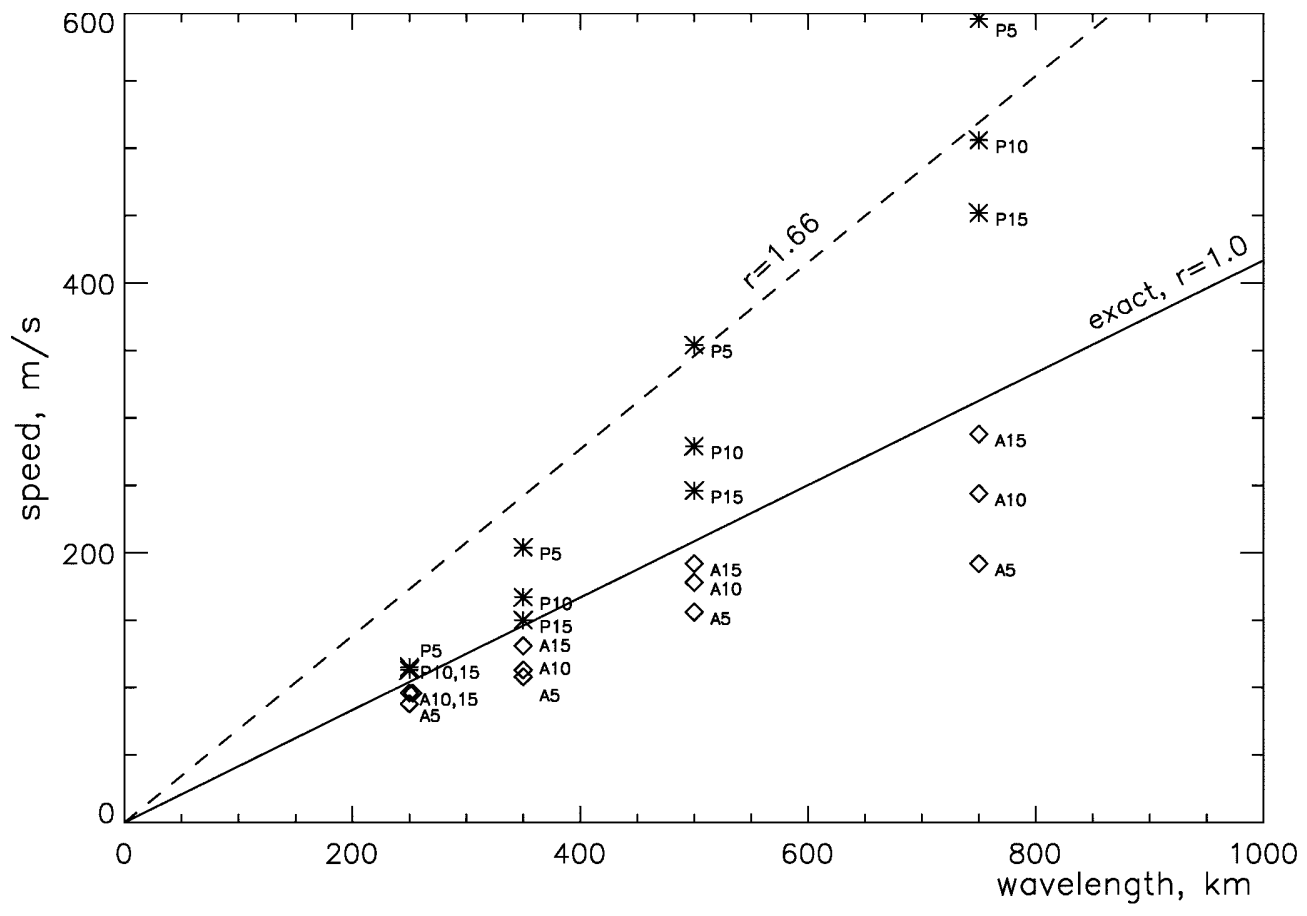

Fig. 3. Summary of the velocities calculated from the simulations. The apparent velocities calculated from the power and elevation angle enhancements, such as the dashed lines superimposed on Plate 2, are plotted. The notation on the plotted symbols tells whether the velocity was calculated from power or angle, and the amplitude of the modeled TID. The correct result is shown by the solid line
TID/gravity waves was fixed at $100 \mathrm{~km}$ which appeared to be suitable for the December conditions around $200 \mathrm{~km}$ for these TIDs. Using a thicker ionosphere and different vertical wavelength gave speed estimations for power that were somewhat different from our simulations. However, the speed derived from the elevation angles did not change greatly, emphasizing the finding that the elevation angle plots give better speed estimations than do the power plots.

If conditions are such that our simulations apply, one can do a further deduction from the data shown in Fig. 3. In Fig. 4B the ratio $(V p-V a) / V c$ is plotted versus wavelength. Although the $250 \mathrm{~km}$ results just show a clustering near 0 , for longer wavelengths the data show a good linear fits as a function of TID amplitude. Therefore in analyzing a TID, one could first determine $V c$ using Eq. (1), then after selecting a suitable period for the TID the wavelength could be calculated, and finally the amplitude of the TID could be calculated from Fig. 4B.

A further property of the TID that one would wish to determine from the observations is the phase. For instance, one would want to determine when the maximum height perturbation of the TID is over some particular location. We tried several possible ways of deducing this from the modeled results, since there does not seem to be any exact way of calculating the TID location. The following procedure gave good results: one carefully examines Fig. 2, it can be seen that the focused rays are reflected from near where the TID has peak height. The figure shows this by indicating, lowest panel, the part of the range-height plot where there is focusing (marked by ' $F$ ' and an arrow). It is seen that the focused rays are at elevation angles of about $14^{\circ}$. In the top panel the ray at $14^{\circ}$ is shown thicker than other rays. It can be seen that the ionospheric reflection of this ray is very near a height maximum of the TID.
Therefore to derive the location of the TID just find the elevation angle for the range at which power focusing occurs at a particular time. From this elevation angle, $\theta_{\mathrm{e}}$, and the ionospheric height the ground distance to the maximum of the TID can be estimated. The main uncertainty is the assumed ionospheric height. Looking at the focused raypath in Fig. 2 it can be seen that there is considerable curvature of the ray in the ionosphere. It appeared that the best ionospheric height to use for the distance calculation would be about $250 \mathrm{~km}$, where the raypath tangent intersects the height of the $F$ region peak of the electron density profile that was used, and well above the actual reflection height of the focused rays. Using this $h_{i o n}$ the ground distance to the TID is then calculated as:

$\mathrm{d}_{\mathrm{TID}}=111^{*}\left[90^{\circ}-\theta_{\mathrm{e}}-\sin ^{-1}\left(\cos \theta_{\mathrm{e}}^{*} 6371 /\left(6371+h_{\text {ion }}\right)\right)\right]$

After calculating the location of the height maximum of the TID at a particular time using Eq. (2), the TID location at other times can be determined using the velocity determined in the earlier part of the TID analysis.

\section{Discussion}

Since the modeling of the TID effects, as described used real data from a particular day for setting many of the parameters, one asks how well did the suggested procedures work for determining the TID properties on this day? Of course we do not know the correct answer for many of the TID properties, such as the speed. For large TIDs it is possible to measure the angular perturbation of the reflection direction as a TID passes over using a digital ionosonde with spaced antennas. From the swings in angle-of-arrival, and 

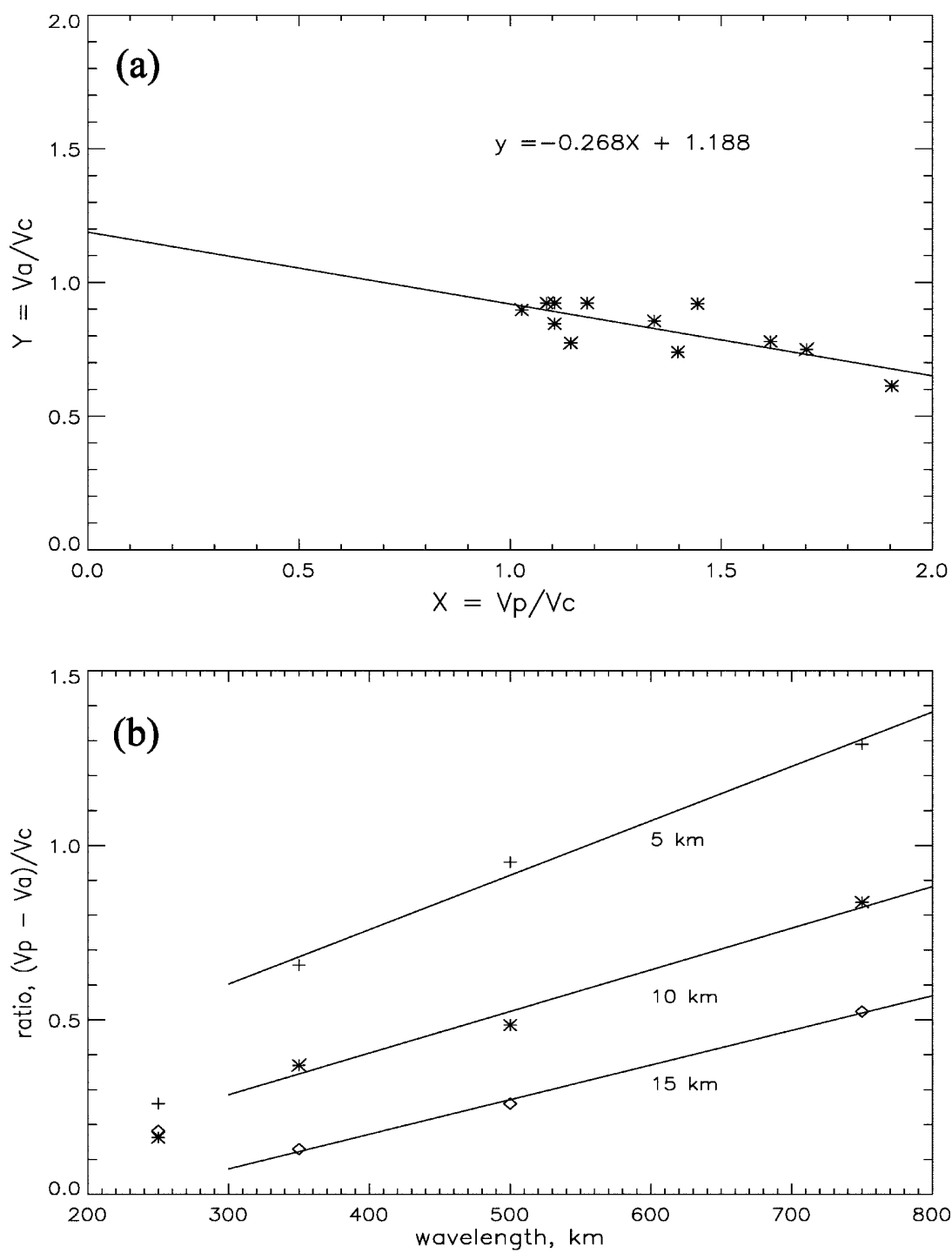

Fig. 4. Derivation of TID properties from the apparent velocities. See text for detail knowing the height perturbation of the TID one can estimate the wavelength, and hence the speed. However, for the 1997 December 6 TIDs, the swings in angle are too small to measure: these are actually relatively weak TIDs. Therefore we have no way of checking our speed estimations. We can check, though, whether the procedure gives reasonable agreement for the TID amplitudes, and whether it correctly gives the times when the TID maxima are over the Rabbit Lake CADI ionosonde.

The comparison results are presented in Table 1. The first results to be compared in this table are the heights (TID amplitudes), calculated by the procedure described above and using Fig. 4B. The calculated heights are shown in column (8) and the heights measured by the CADI Rabbit Lake ionosonde are shown in column (9). It can be seen that there is reasonable agreement of heights except for cases when the TIDs were not clearly defined for purposes of obtaining their apparent velocities. These are shown in brackets.

The second comparison is with the times of TID passage over the Rabbit Lake CADI ionosonde at a range of $680 \mathrm{~km}$. These times are shown in the last two
Table 1. Results of TID analysis for December 61997 using Saskatoon Beam 5

\begin{tabular}{lllllllllll}
\hline$(1)$ & $(2)$ & $(3)$ & $(4)$ & $(5)$ & $(6)$ & $(7)$ & $(8)$ & $(9)$ & $(10)$ & $(11)$ \\
TID & Time & $V p$ & $V a$ & $V c$ & $T$ & $\lambda$ & $\Delta h^{\prime}$ & $\Delta h$ & $T_{680}^{\prime}$ & $T_{680}$ \\
\hline A & $18: 30$ & $(210)$ & 196 & $(213)$ & 43 & $(549)$ & $(\sim 20)$ & 11 & $18: 17$ & $18: 10$ \\
B & $19: 30$ & 228 & $(147)$ & $(176)$ & 46 & $(486)$ & $(9)$ & 9 & $19: 09$ & $19: 10$ \\
C & $20: 00$ & 325 & 176 & 245 & 40 & 588 & 10 & 13 & $19: 54$ & $19: 47$ \\
D & $20: 30$ & 303 & $(126)$ & $(176)$ & 37 & $(390)$ & $(\sim 3)$ & 11 & $20: 24$ & $20: 25$ \\
E & $21: 15$ & 344 & 234 & 276 & 33 & 546 & 13 & 14 & $21: 11$ & $20: 55$ \\
F & $22: 00$ & 344 & 216 & 261 & 23 & 360 & 8 & 7 & $21: 58$ & $22: 00$
\end{tabular}

Less certain values are shown in brackets. Columns (1), (2) identification of the TID cycle; (3), (4), (5) calculated TID speeds from power, angle, and combined using Eq. (1); (6) period for the TID; (7) calculated horizontal wavelength; (8) amplitude of the TID using Fig. 4B; (9) observed amplitude from ionosonde data; (10) calculated time when height maximum of the TID would be at $680 \mathrm{~km}$; (11) observed height maximum time at $680 \mathrm{~km}$

columns; Column (10) shows the calculated times, and column (11) shows the observed times. Note that some of the times agree closely while others have a significant difference. It should be borne in mind that the procedure 
for obtaining the times, shown in column (10), from the observed TID power and angle plots is not a precise calculation but more a procedure that is intended to gives a reasonable approximation. Also TIDs have an observable phase change with height (i.e., a vertical wavelength). The ionosonde times, column (11), used the TID variations observed at $3 \mathrm{MHz}$. At a higher ionosonde frequency (greater height) the times were observably earlier and it is easy to explain the time discrepancies as just being a height effect. Allowing for the imprecise nature of the procedure for deriving the time, and the spread inherent in the time if the TIDs have a finite vertical wavelength, we believe that the suggested procedure provides a useful way of locating the TID in time and space.

\section{Conclusions}

This work shows procedures for deriving TID properties from SuperDARN observations and verifies that these procedures give TID properties that are consistent with measured TID properties. In particular the speeds of the TIDs derived by these procedures are much higher than previous speed estimates. If only range-time-intensity plots are available then often a reasonable estimation of the wave speed would be to make it the same as the apparent wave speed measured from the sloping intensity features.

However, if both RTI plots and elevation angle plots are available then the suggested procedures is:-

1. Calculate apparent velocities, $V p, V a$, from the power and elevation angle SuperDARN plots.

2. From these derive a best fit velocity using Eq. (1) $V c=0.23 \mathrm{Vp}+0.84 \mathrm{Va}$.

3. Derive the TID amplitude using the apparent velocities and Fig. 4B.

4. Estimate the time when a height maximum of the TID is at a particular location by the procedure of measuring the elevation angle, $\theta_{\mathrm{e}}$, of the power maximum and using Eq. (2).
Note that these procedures were derived for winter daytime high-latitude conditions and would need to be modified for other conditions.

Acknowledgement. Topical Editor M. Lester thanks R. Hunsucker and A. Stocker for their help in evaluating this paper.

\section{References}

Bristow, W. A., and R. A. Greenwald, Estimating gravity wave parameters from oblique high-frequency backscatter: modeling and analysis, J. Geophys. Res., 100(A3), 3639-3648, 1995.

Bristow, W. A., R. A. Greenwald, and J. C. Samson, Identification of high-latitude acoustic gravity wave sources using the Goose Bay HF radar, J. Geophys. Res., 79, 319-331, 1994.

Gething, P. J. D., Radio direction finding and superresolution, 2nd edn, Peter Peregrinus, Stevenage, UK, 1991.

Hall, G. E., J. W. MacDougall, J.-F. Cecile, D. R. Moorcroft, and J. P. St.-Maurice, Finding gravity wave source positions using the Super Dual Auroral Radar Network, J. Geophys. Res., 104(A1), 67-78, 1999.

Hazelgrove, J., The Hamiltonian ray path equations, J. Atmos. Terr. Phys., 25, 397-399, 1963.

Hines, C. O., The upper atmosphere in motion, Geophys. Monogr. Ser., 18, AGU, Washington, D.C. 1974.

Hocke, K., and K. Schlegel, A review of atmospheric gravity waves and travelling ionospheric disturbances: 1982-1995, Ann. Geophysicae, 14, 917-940, 1996.

Hunsucker, R. D., Atmospheric gravity waves generated in the high-latitude ionosphere: a review, Rev. Geophys. Space Phys., 20, 293-315, 1982.

Robinson, T. R., Acoustic gravity wave growth and damping in convecting plasma, Ann. Geophysicae, 12, 210-219, 1994.

Samson, J. C., R. A. Greenwald, J. M. Ruohoniemi, and K. B. Baker, High-frequency radar observations of atmospheric gravity waves in the high-latitude ionosphere, Geophys. Res. Lett., 16, 875-878, 1989.

Schanda, E., Physical fundamentals of remote sensing, Springer, Berlin Heidelberg New York, 1986.

Stocker, A. J., N. F. Arnold, and T. B. Jones, The synthesis of travelling ionospheric disturbance (TID) signatures in HF observations using ray tracing, Ann. Geophysicae, 18, 56-64, 2000.

Titheridge, J. E., Ionogram analysis with the generalized program POLAN, Report UAG-93, World Data Center A, Boulder, Co., 1985. 